\title{
Fish Recognition based on HOG Feature Extraction using SVM Prediction
}

\author{
Ms. P. Antony Seba ${ }^{1}$, Ms. S. Rama Subbu Laskhmi ${ }^{2}$, Ms. P. Umamaheswari ${ }^{3}$ \\ Assistant Professor, Kamaraj College of Engineering and Technology ${ }^{1}$ \\ UG Student, Kamaraj College of Engineering and Technology $y^{2,3}$
}

\begin{abstract}
Fish image recognition and prediction is a challenging task. This Paper gives an idea about recognition of fish image using correlation coefficient and then Histogram of oriented Gradient (HOG).Finally the fish images are classified based on their features using Support Vector Machine (SVM).This paper aims to detect which species of fish appears on a fishing boat, based on images captured from boat cameras of various angles and this can be used to prevent fishing of endangered fishes.
\end{abstract}

Keywords: Histogram of oriented Gradient (HOG), Support Vector Machine (SVM), Fish Recognition.

\section{INTRODUCTION}

Recently, a lot of works has been done by depending on the computer; In order to let the processing time to be reduced and to provide more results that are accurate, for example, depending on different types of data, such as digital image and characters.

And an automatic fish image recognition system is proposed in this work. Digital image recognition has been extremely found and studied. Various approaches in image processing and pattern recognition have been developed by scientists and engineers to solve this problem. That is because it has an importance in several fields. In this paper, system for recognizing fish image is built, which may benefit the nature conservancy. The input is considered to be an image of specific size and format; the image is processed and then recognized based on the correlation coefficient and HOG feature extraction

\section{RELATED WORKS}

In Fish Identification System-James Conna[2] lays the foundation for the development of an interactive system that provides the user with instant feedback about a specific fish. A video camera captures a video sequence of fish swims in a tank at the aquarium. The footage is displayed on the screen and the user can click a fish. The system recognizes the fish species and displays the recognition result to the user. The system was tested on a total of 25 fish and achieved an overall recognition accuracy of $88.6 \%$ across all fish. The selected fish is segmented within the image and then recognized. It provides the instant feedback about a specific fish.

In Fish Detection Solution Using Neural Network Approach-Mustafa Man[3], used neural network to recognizes the fish object from a small window that scans for the object all over the image. The neural network will examine the windows of the image and decides whether each window contains a fish. This approach is aims to eliminate the difficult task of manually selecting the fishes, which must be chosen and localized to be analyzed. This work is a part of process on detecting the fish, counting and estimating the length of the fish. Their paper presents a neural networkbased approach for detecting fish object inside a digital image. Also they aimed to eliminate the difficult task of manually selecting the fishes. Fish Recognition Based On Robust Features-Ibrahim Almarashdeh[1], started by acquiring an image containing pattern of fish, then the image segmentation is performed relying on color texture measurements. To recognize an isolated pattern of interest in the image based on the combination between robust features extraction. Where depend on color texture measurements that are extracted by gray level co-occurrence matrix. They only concerned about color textures.

\section{PROPOSED SYSTEM}

This system can be used for recognition of fish image using correlation coefficient and then Histogram of oriented Gradient (HOG).Finally the fish images are classified based on their features using Support Vector Machine(SVM) as shown in Fig.3d 


\section{TEMPLATE MATCHING}

Template matching is a technique in digital image processing for finding small parts of an image which match a template image. It can be used in manufacturing as a part of quality control, a way to navigate a mobile robot, or as a way to detect edges in images. Before matching the image should be converted to gray scale as shown in Fig 3 a.
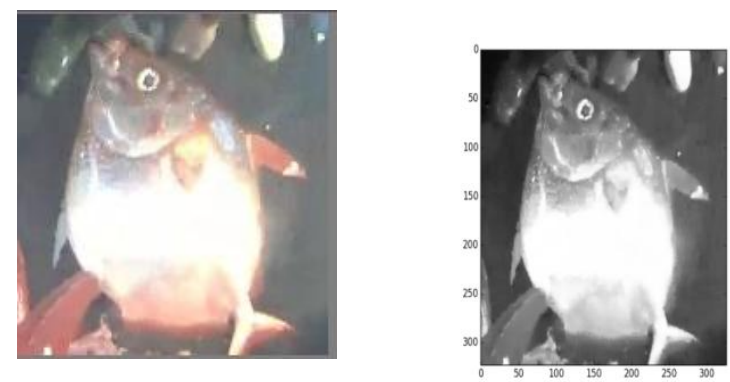

Fig.3a: Template and Gray Image

Then correlation coefficient is applied for matching to the input image as shown in Fig.3b.

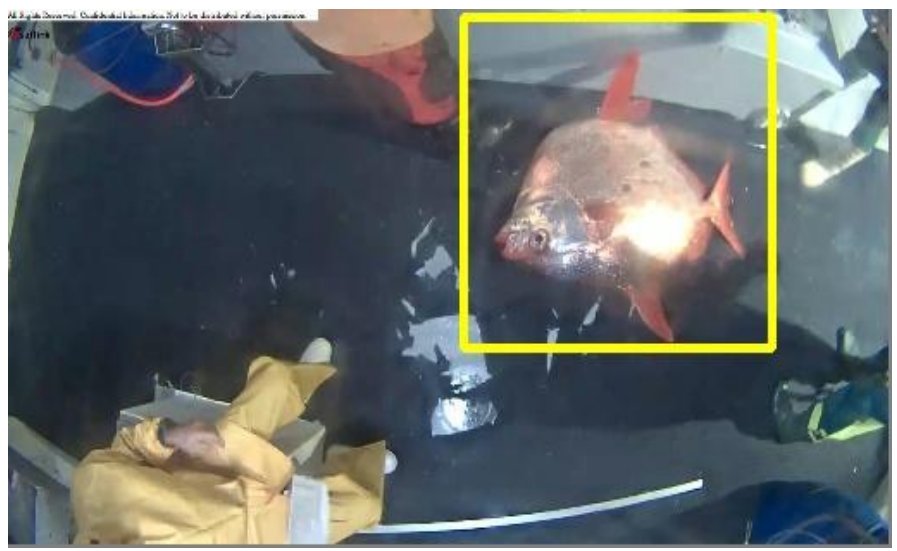

Fig 3c.Template Matching for input image

\section{HOG FEATURE EXRATION}

The Histogram of Orientation Gradients (HOG) is a feature descriptor used in computer vision and image processing for the purpose of object detection. The Fig.3c shows the HOG gradients for the template image. The technique counts occurrences of gradient orientation in localized portions of an image. This method is similar to that of edge orientation histograms, scale-invariant feature transform descriptors, and shape contexts, but differs in that it is computed on a dense grid of uniformly spaced cells and uses overlapping local contrast normalization for improved for improved accuracy.

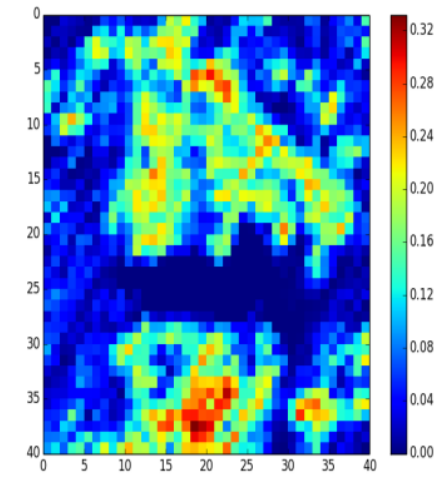

Fig.3c.HOG features for template image

The cropped image from the template matched input image and the HOG gradients are in Fig. $3 \mathrm{~d}$. 

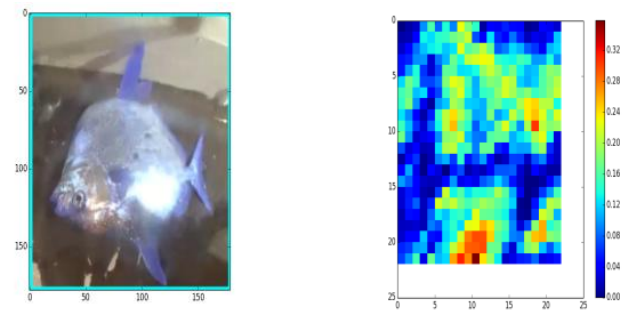

Fig. 3d.HOG feature for test template

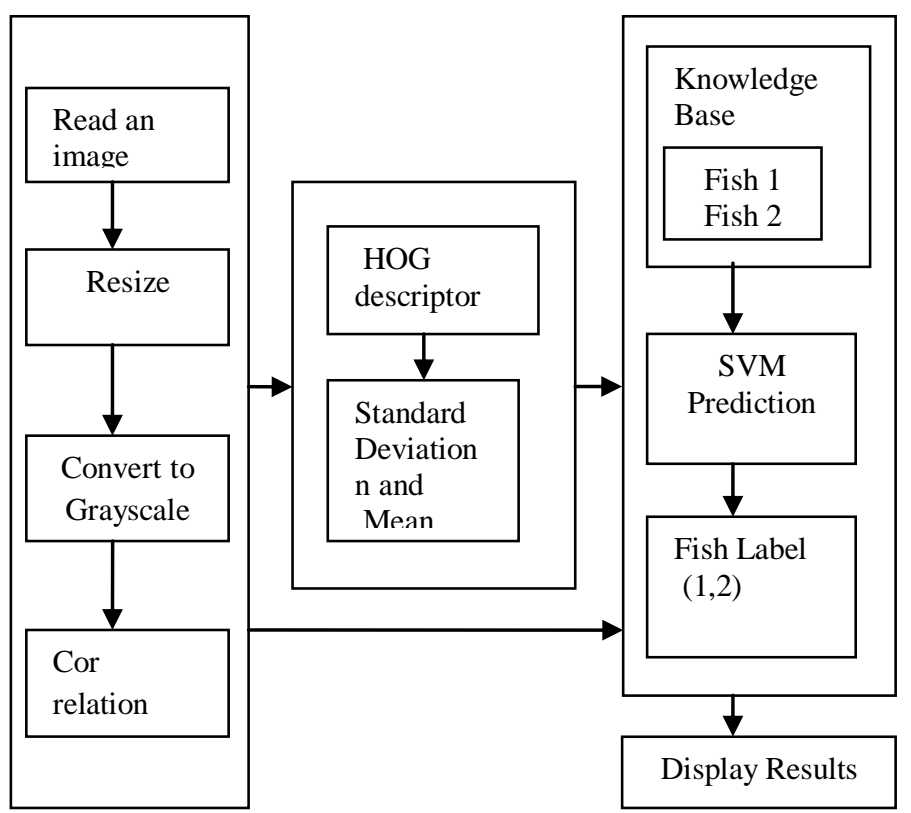

Fig.3d Proposed System for fish recognition and classification.

\section{CLASSIFICATION}

Classification and prediction are two forms of data analysis that can be used to extract models describing important data classes or to predict future data trends. Such analysis can help provide us with a better understanding of the data at large. Whereas classification predicts categorical (discrete, unordered) labels, prediction models continuous valued functions.
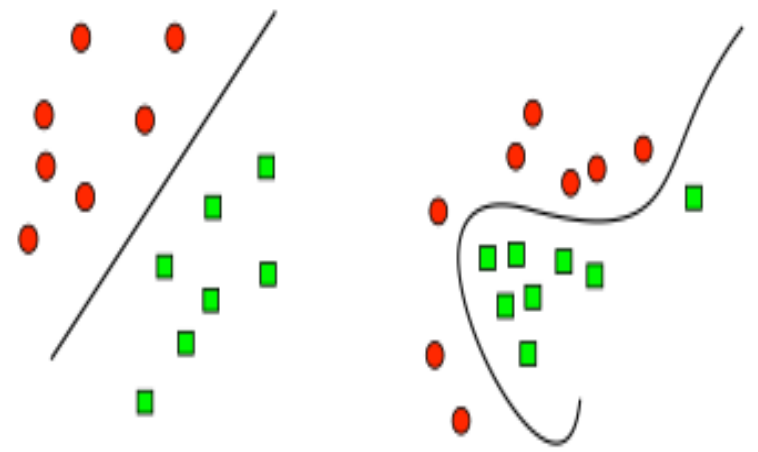

Figure 3. (a) Linear classification; 3.(b) Non-linear classification.

\section{RESULTS}

The number of images for training set is 72 and the test set is 18 .

The receiver operating characteristic curve(ROC) is found by applying HOG feature on images and then calculating the standard deviation and mean,the area is found to be 45\%. as shown in Fig.4a.The ROC area by combining correlation coefficient and HOG is found to be $94 \%$ as shown in Fig.4b. 


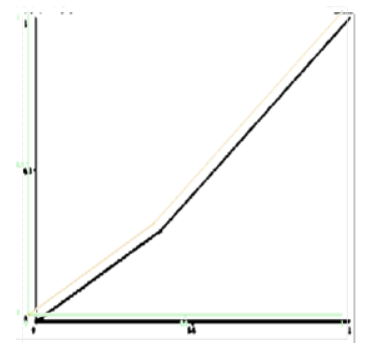

TUNA

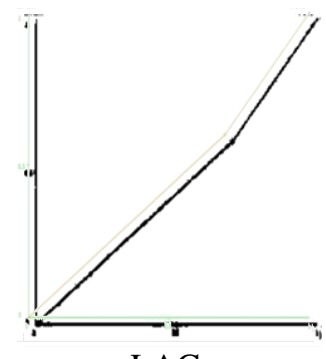

LAG

Fig.4a. ROC for HOG

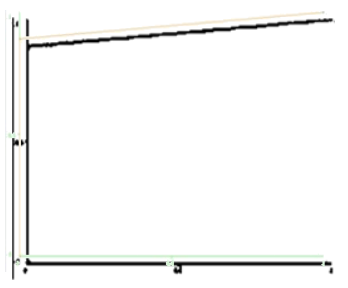

TUNA

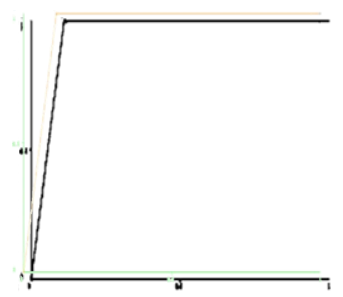

LAG

Fig 4b. ROC for CCOEFF_NORMED and HOG

\section{CONCLUSION AND FUTURE ENHANCEMENTS}

We have obtained a very good results by combining the correlation coefficient and HOG features. The fish dataset possess tampered fishes, which is very challenging in identifying the species. Therefore in future we would like apply geometric features identify the partial tampered images.

\section{REFERENCES}

[1] Ibrahim Almarashdeh ,A,(2010)," Fish Recognition Based On Robust Features", on Journal of Theoretical and Applied Information Technology.

[2] James Connan , H,(2016)," Fish Identification System”, IEEE Transactions on Pattern Analysis and Machine Intelligence 20 (2010)

[3] Mustafa Man, K, (2011), "Fish Detection Solution Using Neural Network Approach", IEEE Transaction on Neural Network, Vol.2.

[4] Saitoh ,T,(2016)," Feature Points based Fish Image Recognition”, on InterNational Journal of Computer Information Systems and Industrial Management Application, ISSN 2150-7988 Vol.8.

[5] Takeshi Saitoh ,S ,(2014)," An Overview on Template Matching Methodologies and its Applications”, on International Conference on Matching Templates. 